\title{
Anchoring tick salivary anti-complement proteins IRAC I and IRAC II to membrane increases their immunogenicity
}

\author{
Laurent GILlet $^{1}$, Hélène Schroeder ${ }^{1}$, Jan MAST $^{2}$, Muriel Thirion ${ }^{1}$, \\ Jean-Christophe Renauld ${ }^{3}$, Benjamin Dewals ${ }^{1}$, Alain VAnderplasschen ${ }^{1 *}$ \\ ${ }^{1}$ Immunology-Vaccinology (B43b), Department of Infectious and Parasitic Diseases (B43b), \\ Faculty of Veterinary Medicine, University of Liège, B-4000 Liège, Belgium \\ ${ }^{2}$ Department Biocontrole, Research Unit Electron Microscopy, Veterinary and Agrochemical Research Centre, \\ VAR-CODA-CERVA, Groeselenberg 99, B-1180 Ukkel, Belgium \\ ${ }^{3}$ Ludwig Institute for Cancer Research, Brussels Branch, and Experimental Medicine Unit, \\ Université de Louvain, Brussels, Belgium
}

(Received 25 November 2008; accepted 16 June 2009)

\begin{abstract}
Tick salivary proteins are promising targets for the development of anti-tick vaccines. Recently, we described two paralogous anti-complement proteins, called Ixodes ricinus anti-complement (IRAC) proteins I and II, that are co-expressed in tick I. ricinus salivary glands. However, our previous attempts to immunize rabbits against IRAC via infection with recombinant Bovine herpesvirus 4 (BoHV-4) vectors invariably failed although both recombinants expressed high levels of functional IRAC proteins in vitro. As IRAC are soluble monovalent antigens, one of the possible explanations is that monovalent ligation of the B-cell receptor induces receptor activation but fails to promote antigen presentation, a phenomenon that is thought to induce a state of B-cell tolerance. In the present study, we tried to increase IRAC immunogenicity by expressing them as oligovalent antigens. To this end, IRAC were fused to membrane anchors and BoHV-4 vectors expressing these recombinant forms were produced. The immunization potentials of recombinant viruses expressing either secreted or transmembrane IRAC proteins were then compared. While the former did not induce a detectable immune response against IRAC, the latter led to high titres of anti-IRAC antibodies that only marginally affected tick blood feeding. All together, the data presented in this study demonstrate that the immunogenicity of a soluble antigen can be greatly improved by anchoring it in membrane.
\end{abstract}

BoHV-4 / IRAC / tick salivary antigen / antigen valency

\section{INTRODUCTION}

Ticks are blood-feeding ectoparasites. Several observations suggest that inhibition of the host complement alternative pathway is crucial for the achievement of tick blood feeding. First, it has been demonstrated that the saliva of several Ixodes species contains an inhibitor of the alternative pathway [17, 24]. Second, the host

\footnotetext{
* Corresponding author: A.vdplasschen@ulg.ac.be
}

range of Ixodes ticks correlates with their ability to counteract the alternative pathway of their most common host species [16]. Third, cases of host resistance to ticks have been described in which the complement system is implicated with a predominant part of the alternative pathway $[16,24,30]$.

Recently, we described two novel salivary anti-complement protein in Ixodes ricinus ticks, termed I. ricinus anti-complement (IRAC) proteins I and II [4]. Both proteins inhibit the

This is an Open Access article distributed under the terms of the Creative Commons Attribution-Noncommercial License (http://creativecommons.org/licenses/by-nc/3.0/), which permits unrestricted use, distribution, and reproduction in any noncommercial medium, provided the original work is properly cited. 
alternative pathway of the human complement by mechanisms similar to the one described for the Ixodes scapularis anti-complement protein Isac [28]. Phylogenetic analyses of IRAC sequences together with other Isac homologues demonstrated that ticks belonging to the I. ricinus complex encode a family of relatively small anti-complement molecules. Indeed, we demonstrated that IRAC I and IRAC II are the products of paralogous genes co-expressed in I. ricinus salivary glands [4]. Phylogenetic analysis revealed that IRAC I and IRAC II sequences were undergoing diversification by a process of positive Darwinian selection and we demonstrated that this diversification through evolution of IRAC I and IRAC II has led to molecules with different inhibitory activities against the complement of different host species [25]. Based on these observations, we made the hypothesis that IRAC I and II, like other tick salivary glands antigens, could be good candidates for the development of antitick vaccines.

Several studies demonstrated that Bovine herpesvirus 4 (BoHV-4) can be used as an efficient in vitro expression vector $[6,9]$. BoHV-4 belongs to the Herpesviridae family, $\gamma$-herpesvirinae subfamily, Rhadinovirus genus [31]. BoHV-4 is not associated with a specific pathology and can be highly prevalent in the cattle population [26]. BoHV-4 infects monocytes in vivo [18] and ex vivo ${ }^{1}$ and these cells are probably the site of viral latency. Manipulations of BoHV-4 genome have recently been facilitated by cloning BoHV-4 as a Bacterial Artificial Chromosome (BAC). Using BAC technology, BoHV-4 recombinants expressing functional IRAC I or II were produced [9] and the vaccine potential of these recombinants for IRAC immunization was tested in vivo using the rabbit model. Surprisingly, despite several attempts, we were not able to induce a humoral immune response against IRAC in vaccinated rabbits (unpublished data).

The failure to induce anti-IRAC antibodies could have several explanations that are not mutually exclusive. It could be the consequence of a too low antigenic concentration or due to

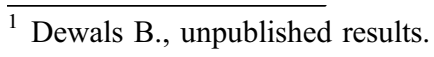

the absence of rabbit T epitopes in IRAC molecules. One additional explanation could rely on the expression of soluble IRAC as monomeric antigen. Indeed, it has recently been shown that antigen-specific B cells have a reduced ability to present monomeric antigens compared to oligovalent forms [14]. It was suggested that this phenomenon could explain the tolerogenic properties of some monovalent antigen preparations.

In the present study, we addressed the feasibility to express IRAC as oligovalent antigens on cell surface by anchoring them in membrane. Firstly, IRAC I and II sequences were fused to the transmembrane regions of vaccinia hemagglutinin (HA) or human CD46. These two anchors proved to be appropriate for expression of IRAC as transmembrane fusion proteins. BoHV-4 recombinants expressing IRAC I and II fused to HA or CD46 sequences were then produced using BAC cloning technology. In vitro and ex vivo infections performed with these viruses demonstrated cell surface expression of IRAC. Finally, we compared the potential of BoHV-4 recombinants expressing either soluble or membrane anchored IRAC proteins as anti-IRAC vaccines using the rabbit model. While the former did not induce a detectable immune response against IRAC, the latter led to high titers of anti-IRAC antibodies that partially affected tick blood feeding.

\section{MATERIALS AND METHODS}

\subsection{Ticks and animals}

I. ricinus adult ticks were obtained from the University of Neuchâtel (Switzerland) Tick Rearing Facility. Specific-pathogen-free New Zealand white rabbits were used for the immunization studies and challenge infestation. All experimental protocols were approved by the Institutional Animal Care and Use Committee of the University of Liège (Belgium).

\subsection{Viruses and cell lines}

Madin-Darby bovine kidney (MDBK, ATCC CCL-22), EBL NLS-Cre cells [9] and CrandellReese feline kidney cells (CRFK, ATCC CCL-94) 
Table I. Primers used in this study.

\begin{tabular}{lc}
\hline Primer name & Sequence \\
\hline IRAC I start & 5'-AAAACGGATCCATGAAGACTGCGCTGACC-3 $^{\prime{ }^{\prime}}$ \\
IRAC I $\Delta$ stop & $5^{\prime}$-AAACTCGAGCCGCGGTGGGATGGCCTCAGG-3' \\
IRAC II start & $5^{\prime}$-AAAACGGATCCATGAGGACTGCGCTGACC-3' \\
IRAC II $\Delta$ stop & $5^{\prime}$-AAACTCGAGCCGCGGGGCGATGGCCTCAAG-3' \\
TMHA start & $5^{\prime}$-TCATCCGCGGACCAAGGACTTTGTAGAAA-3' \\
TMHA stop & $5^{\prime}$-TTCTCGAGCTAGACTTTGTTCTCTGTTTTG-3' \\
TMCD46 start & $5^{\prime}$-GCCGCGGATACTTGACAGTTTGGATGTTTG-3' \\
TMCD46 stop & $5^{\prime}$-AAAACTCGAGTCAGCCTCTCTGCTCTGC-3' \\
\hline
\end{tabular}

${ }^{a}$ Bold: BamHI, italic: XhoI, italic and underlined: SacII.

were cultured in minimum essential medium (MEM, Invitrogen, Carlsbald, USA) containing 10\% heatinactivated fetal calf serum (v/v) (FCS, Lonza, Basel, Switzerland). The BoHV-4 V. test strain was used throughout this study.

\subsection{Construction of plasmids encoding membrane anchored forms of IRAC}

IRAC I and II open reading frames (ORF) with exception of the stop codon were amplified by PCR using IRAC I or II start primers and IRAC I or II $\Delta$ stop primers, respectively (Tab. I). The PCR products were then digested with $\mathrm{BamHI}$ and $\mathrm{XhoI}$ and inserted into the $\mathrm{pCDNA}^{\mathrm{TM}} 4 / \mathrm{TO}$ vector (Invitrogen, Carlsbald, USA) opened by the same enzymes, resulting in $\mathrm{pcDNA}^{\mathrm{TM}} 4 / \mathrm{TO}$ IRAC I $\Delta$ stop and $\mathrm{pcDNA}^{\mathrm{TM}} 4 / \mathrm{TO}$ IRAC II $\Delta$ stop. Membrane anchoring regions and stop codon were amplified from Vaccinia HA (TMHA) (GenBank AY243312, amino-acid residues 274 to 315 ) and human CD46 (TMCD46) (GenBank NM_172361, amino-acid residues 294 to 355) using the primers listed in Table I. PCR products were then digested with $\mathrm{SacII}$ and $X h o$ I and inserted into pcDNA ${ }^{\mathrm{TM}} 4 / \mathrm{TO}$ IRAC I $\Delta$ stop and $\mathrm{pcDNA}^{\mathrm{TM}} 4 / \mathrm{TO}$ IRAC II $\Delta$ stop opened by the same enzymes, resulting in pcDNA ${ }^{\text {TM }} 4 / T O$ IRAC I $\mathrm{HA}, \mathrm{pcDNA}^{\mathrm{TM}} 4 / \mathrm{TO}$ IRAC I CD46, $\mathrm{pcDNA}^{\mathrm{TM}} 4 / \mathrm{TO}$ IRAC II HA, and pcDNA ${ }^{\mathrm{TM}} 4 / \mathrm{TO}$ IRAC II CD46.

\subsection{Viral mutagenesis}

Mutagenesis of wild-type V. test BAC G plasmid [9] was performed by a two-step mutagenesis procedure in bacteria using the shuttle plasmid pST76KSR-lacZ [9]. Expression cassettes were inserted in the far right $B s t$ EII site of BoHV-4
L-DNA (Fig. 1A) as performed before for cassettes encoding soluble forms of IRAC proteins [9]. Plasmids to induce homologous recombination were constructed as follows. PcDNA ${ }^{\mathrm{TM}} 4 / \mathrm{TO}$ IRAC I HA, pcDNA $^{\mathrm{TM}} 4 /$ TO IRAC I CD46, pcDNA ${ }^{\mathrm{TM}} 4 /$ TO IRAC II $\mathrm{HA}$ and $\mathrm{pcDNA}^{\mathrm{TM}} 4 / \mathrm{TO}$ IRAC II CD46 were digested with PmeI and the appropriate fragments were inserted into the pST76KSR-lacZ IRAC I or II digested with PmeI, resulting in pST76KSR-lacZ IRAC I HA, I CD46, II HA, II CD46.

\subsection{Southern blotting}

Southern blot analysis was performed as described previously [20].

\subsection{Antibodies}

Monoclonal antibodies (mAbs) directed against rabbit CD11b (198), IgM (NRBM) and CD5 (KEN-5) were purchased from Serotec (Oxford, UK) and used according to the manufacturer recommendations. Anti-IRAC I and anti-IRAC II mAbs [4] were also used in this study.

\subsection{Indirect immunofluorescent staining of adherent cells}

MDBK infected cells and CRFK transfected cells were fixed in PBS containing 4\% (w/v) paraformaldehyde (Sigma-Aldrich, St. Louis, USA) for $10 \mathrm{~min}$ on ice and then for $20 \mathrm{~min}$ at $20^{\circ} \mathrm{C}$. After washing with PBS, samples were permeabilized in PBS containing $0.1 \%$ (w/v) NP-40 (Sigma-Aldrich) at $37{ }^{\circ} \mathrm{C}$ for $10 \mathrm{~min}$. Immunofluorescent staining (incubation and washes) was performed in PBS containing $10 \%$ FCS (v/v). Samples were incubated at $37^{\circ} \mathrm{C}$ for $45 \mathrm{~min}$ 


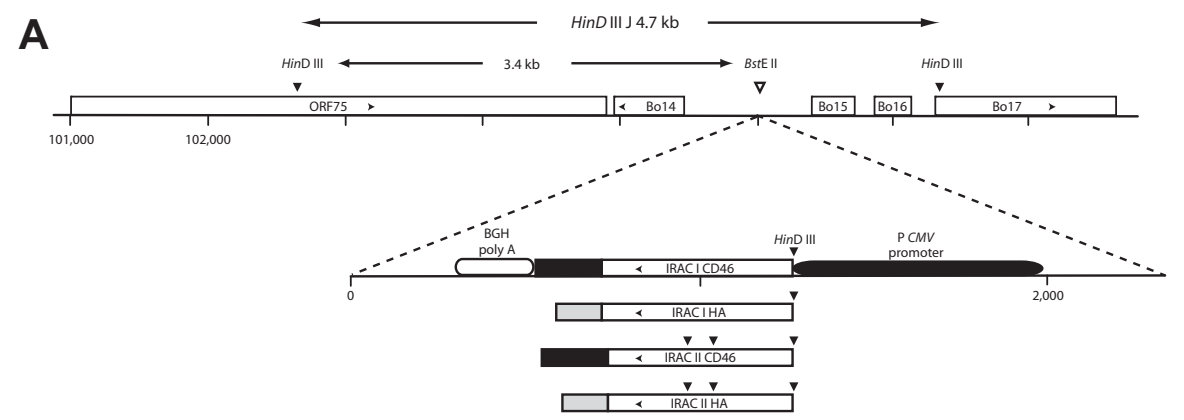

B
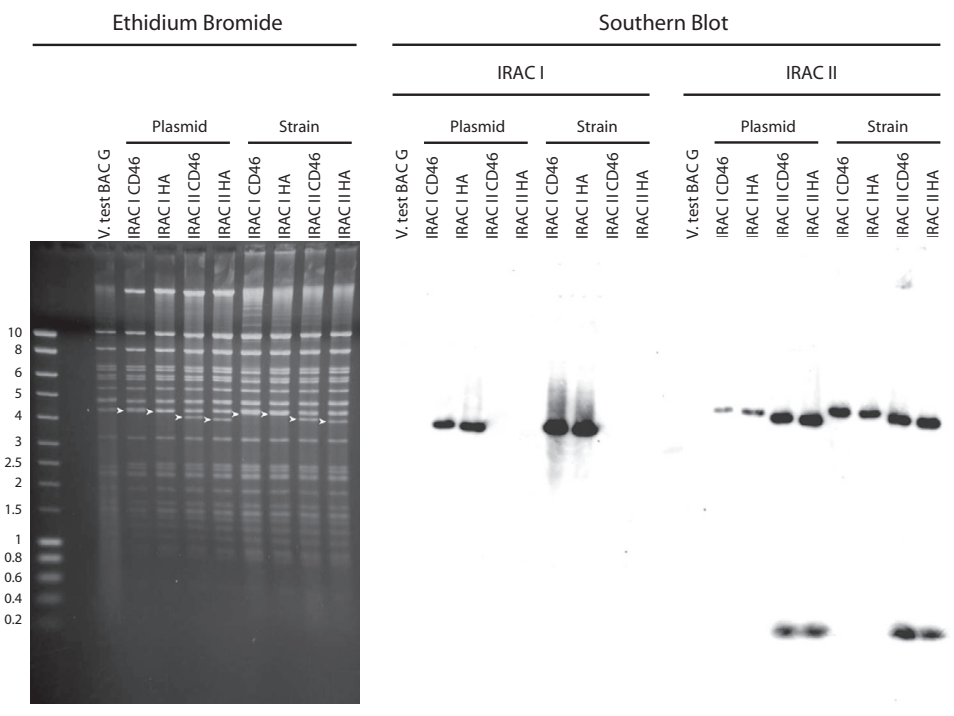

C

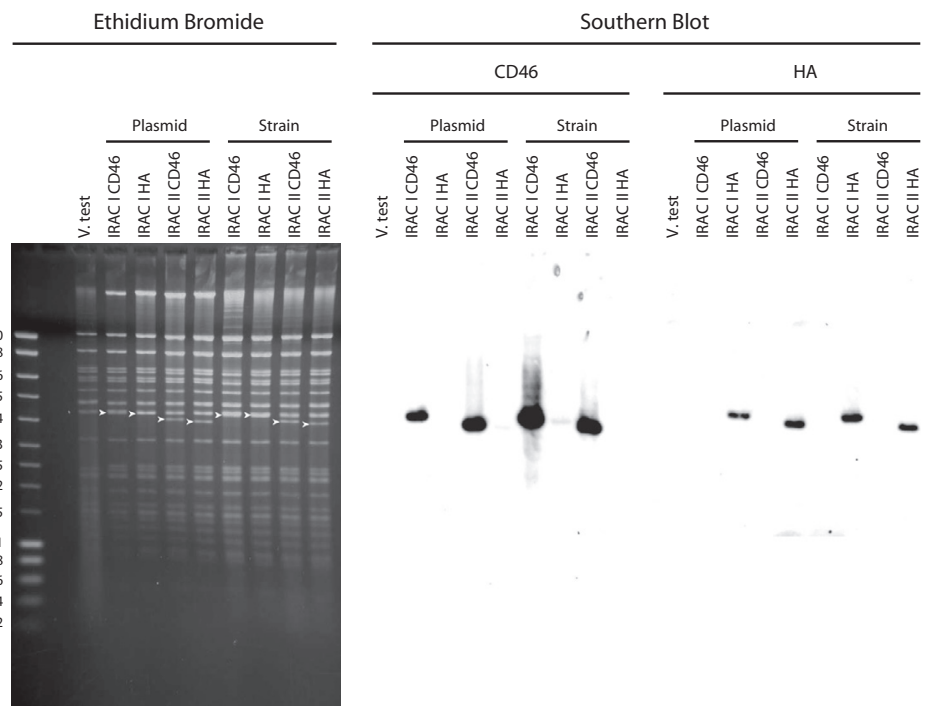


with different mAbs (Figs. 2-4) or dilutions of rabbit serum (Fig. 5). After three washes, samples were incubated at $37{ }^{\circ} \mathrm{C}$ for 30 min with Alexa Fluor 488 goat anti-mouse (GAM) IgG $(2 \mu \mathrm{g} / \mathrm{mL}$; Invitrogen) or Alexa Fluor 488 goat anti-rabbit $\operatorname{IgG}(2 \mu \mathrm{g} / \mathrm{mL}$; Invitrogen).

\subsection{Peripheral blood mononuclear cells isolation and staining}

Peripheral blood mononuclear cells (PBMC) were prepared as described elsewhere [11]. Cells were washed extensively with PBS before use. Immunofluorescent labeling was performed in PBS containing $10 \%$ FCS. For cell phenotyping, living cells were incubated on ice for $30 \mathrm{~min}$ with the mAbs described above as the primary antibody. Samples were then washed with PBS and incubated on ice for $20 \mathrm{~min}$ with Alexa Fluor 633 conjugated goat anti-mouse IgG $(\mathrm{H}+\mathrm{L})$ (Alexa 633-GAM, $5 \mu \mathrm{g} / \mathrm{mL}$; Invitrogen) as the secondary antibody.

\subsection{Microscopy analysis}

Epifluorescent microscopy analysis was performed with a DMIRBE microscope (Leica, Wetzlar, Germany) equipped with a DC 300F CCD camera (Leica) as described previously [5].

\subsection{Flow cytometry}

Flow cytometry acquisitions and analyses were performed using a three-laser Becton Dickinson fluorescence-activated cell sorter (FACSAria) [10] (Becton Dickinson, Erembodeghem, Belgium).

\subsection{Immunization}

Five groups, each consisting of two rabbits, were used in this study. Animals from the different groups were injected intravenously 2 times at one month interval with $10^{8}$ PFU of BoHV-4 V. test WT, V. test BAC G IRAC I, V. test BAC G IRAC II, V. test BAC G IRAC I HA or V. test BAC G IRAC II HA excised recombinant strains.

\subsection{Anti complement alternative pathway assay}

The property of IRAC molecules to inhibit the alternative pathway of the rabbit complement system was tested using the AH50 assay as described previously [9]. This assay relied on the lysis of unsensitized human erythrocytes by diluted rabbit serum in conditions that prevent activation of the classical pathway. Briefly, MDBK cells grown in $75 \mathrm{~cm}^{2}$ flask were infected at a MOI of 1 PFU/cell with BoHV-4 V. test, V. test BAC G IRAC I Excised and V. test BAC G IRAC II Excised strains. Forty-eight hours after infection, cell culture supernatants were collected and concentrated. The property of concentrated cell supernatants to inhibit the complement alternative pathway was assayed by addition of various amounts of concentrated cell supernatant to the test.

\subsection{Challenge infestation}

Four weeks after the second immunization, vaccinated and control rabbits were infested with 16 I. ricinus adult couples per rabbit. Ticks were confined in plastic capsule glued with a beewax/colophane mixture on the shaven back [21]. Animals

Figure 1. Production and characterization of BoHV-4 recombinant strains expressing membrane anchored recombinant forms of IRAC I and IRAC II. (A) IRAC I CD46, -I HA, -II CD46 or -II HA expression cassette were inserted in the BstEII site of the V. test BAC G plasmid corresponding to the far left BstEII site of BoHV-4 L-DNA. The insertion was performed by mutagenesis of the V. test BAC G plasmid in bacteria through homologous recombination with pST76KSR-lacZ IRAC I HA, -I CD46, -II HA or -II CD46 plasmids. (B and C) Characterization of BoHV-4 recombinant strains expressing membrane anchored IRAC by a combined restriction endonuclease-Southern blot approach. The DNA of the modified BAC plasmids generated in bacteria or from the reconstituted viral strains were analysed by HindIII restriction (B and $\mathrm{C}$, left panels) and further tested by Southern blot using IRAC I ORF (B, middle panel), IRAC II ORF (B, right panel), human CD46 ORF (C, middle panel) and Vaccinia HA ORF (C, right panel) as probes. Arrows indicate the restriction fragments containing IRAC ORF. Marker sizes (MS) in kb are indicated on the left. 

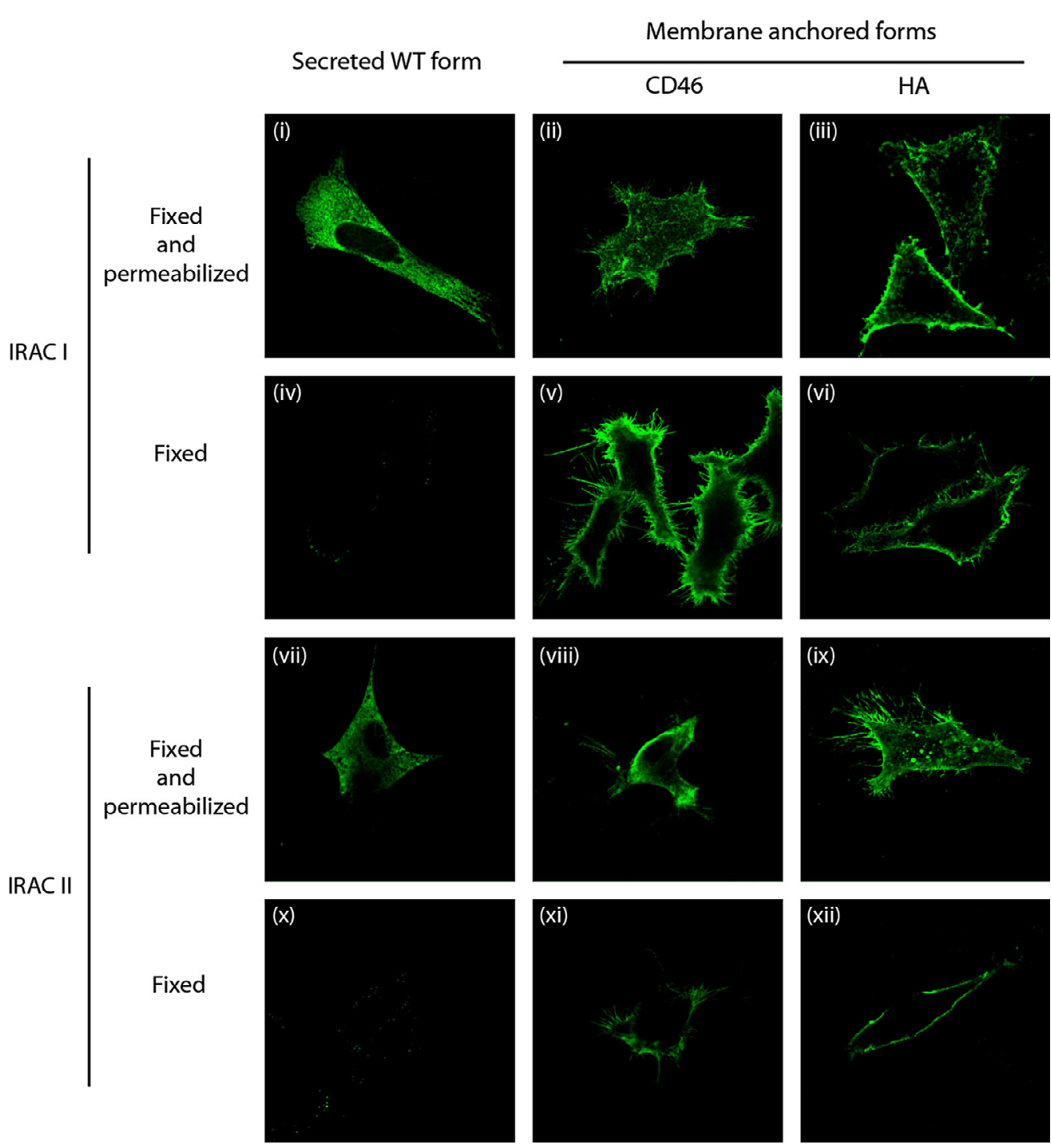

Figure 2. Characterization of membrane anchored recombinant forms of IRAC I and IRAC II. CRFK cells were transfected with $\mathrm{pcDNA}^{\mathrm{TM}} 4 / \mathrm{TO}$ derived vectors encoding the different membrane anchored recombinant forms of IRAC. After $18 \mathrm{~h}$, cells were fixed and permeabilized (panels (i) to (iii) and (vii) to (ix)) or only fixed (panels (iv) to (vi) and (x) to (xii)), then treated as described in the methods for indirect immunofluorescent labeling. mAbs anti-IRAC I or II were used as primary antibodies and were revealed by Alexa488-GAM secondary antibody. The side of each panel corresponds to $30 \mu \mathrm{m}$ of the specimen. (For a color version of this figure, please consult www.vetres.org.)

were monitored two times per day for signs of discomfort or skin lesions, and ticks were counted and examined for attachment. The unattached ticks were counted and removed $24 \mathrm{~h}$ after infestation, and detached engorged ticks were collected daily from each rabbit, counted and weighted. The engorged 


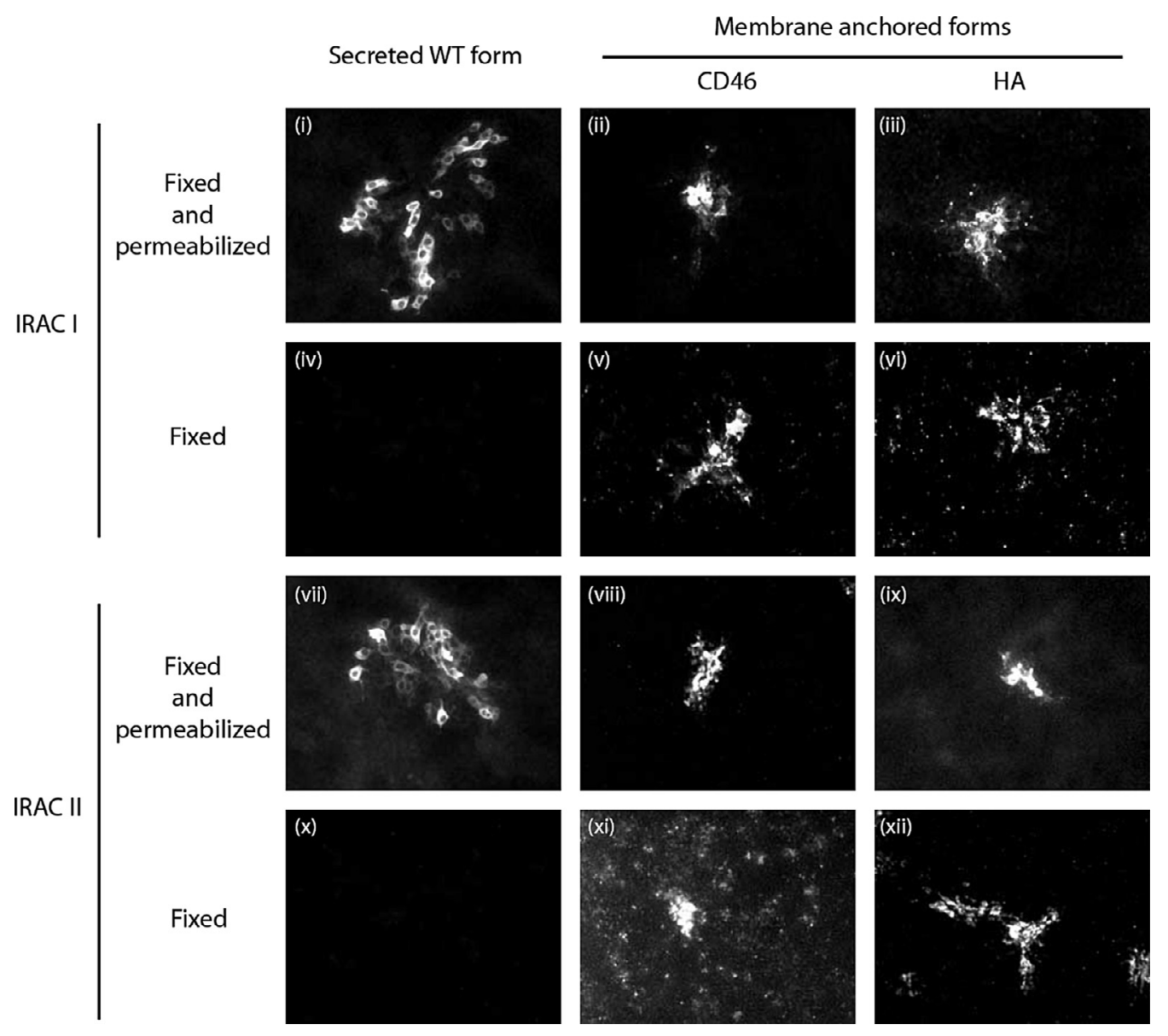

Figure 3. Immunodetection of IRAC expressed by BoHV-4 recombinant strains. MDBK cells were infected at a MOI of $0.01 \mathrm{PFU} /$ cell with BoHV-4 V. test IRAC I (i) and (iv), BoHV-4 V. test IRAC II (vii) and (x), BoHV-4 V. test BAC G IRAC I CD46 (ii) and (v), V. test BAC G IRAC I HA (iii) and (vi), V. test BAC G IRAC II CD46 (viii) and (xi) and V. test BAC G IRAC II HA (ix) and (xii) excised strains. After $48 \mathrm{~h}$, cells were fixed and permeabilized (panels (i-iii) and (vii-ix)) or only fixed (panels (iv-vi) and (x-xii)), then treated as described in the methods for indirect immunofluorescent labeling. mAbs anti-IRAC I or II were used as primary antibodies and were revealed by Alexa488-GAM secondary antibodies. The side of each panel corresponds to $500 \mu \mathrm{m}$ of the specimen.

ticks were held in a humidity chamber $\left(25^{\circ} \mathrm{C} ; 85 \%\right.$ of humidity) for 40 days to evaluate oviposition.

\subsection{Statistical analysis}

Logrank test was used for statistical analysis of data using the program GraphPadPrism 4 (Prism software, Irvine, USA). Statistical significance was determined at $p<0.05$.

\section{RESULTS}

\subsection{Expression of IRAC proteins as membrane anchored recombinant forms}

To express IRAC as oligovalent antigens on the surface of expressing cells, we anchored them in membrane by fusing their C-terminal end to the membrane anchoring regions of 


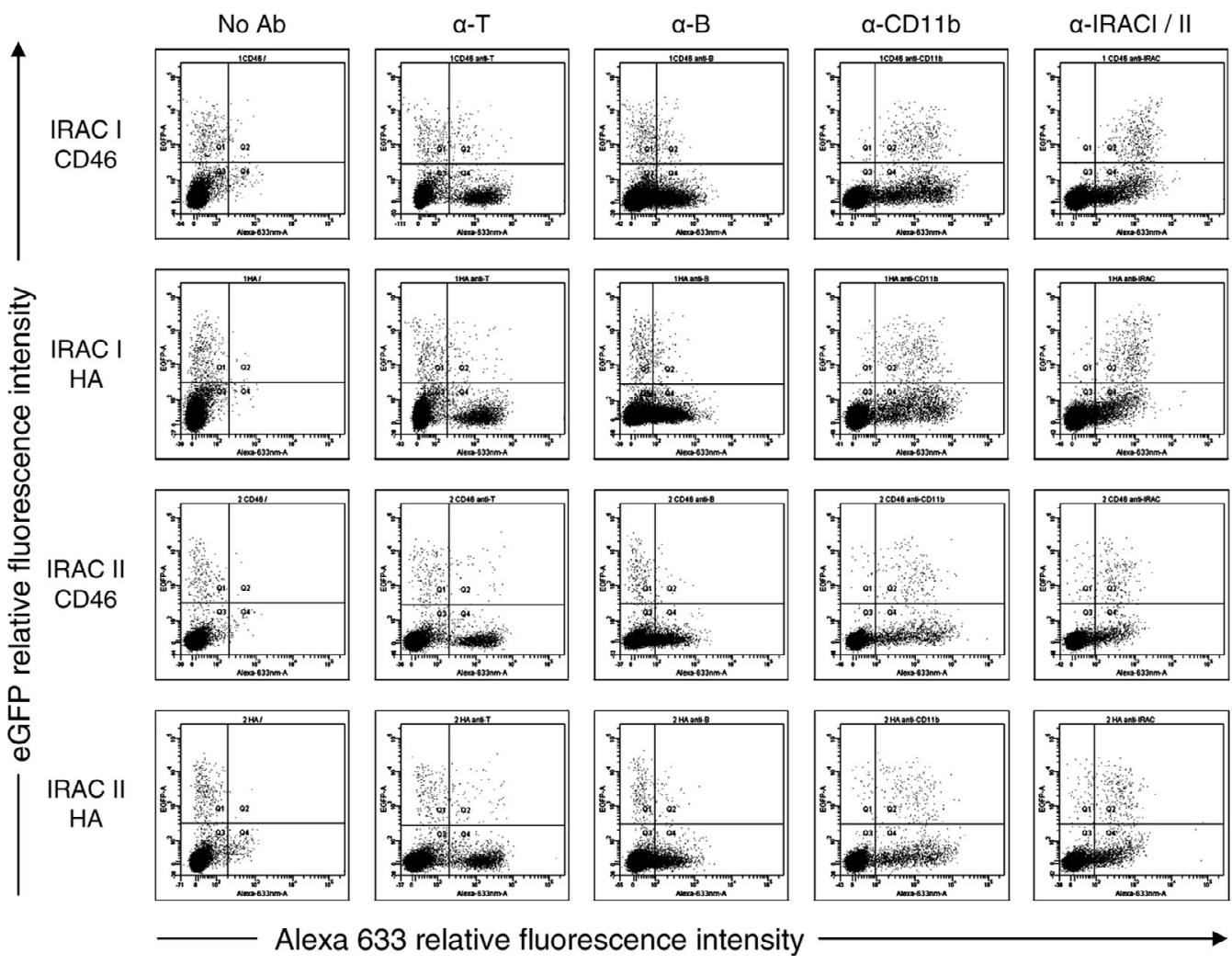

Figure 4. Membrane anchored forms of IRAC proteins are detectable at the surface of PBMC infected ex vivo. Rabbit PBMC were collected as described in the methods and infected at a MOI of $1 \mathrm{PFU} / \mathrm{cell}$ with BoHV-4 V. test BAC G IRAC I CD46, V. test BAC G IRAC I HA, V. test BAC G IRAC II CD46 or V. test BAC G IRAC II HA strains. After $48 \mathrm{~h}$, cells were treated as described in the methods for indirect immunofluorescent labeling with mAbs directed against rabbit CD11b, rabbit IgMs $(\alpha-B)$ or rabbit CD5 $(\alpha-T)$ and then analyzed by flow cytometry for eGFP and Alexa-633 fluorescences.

different proteins as described in the Materials and methods. Two types of anchoring regions were tested: (i) the transmembrane domains of human CD46, hereafter called "CD46" anchor, and (ii) the transmembrane domains of Vaccinia virus WR strain HA, hereafter called "HA" anchor. Immunofluorescent staining of cells transfected with the different plasmids revealed that all recombinant proteins were associated with cell membranes (Fig. 2). Immunofluorescent staining of fixed cells revealed that independently of the kind of anchor, all recombinants proteins were expressed on the cell surface (Fig. 2, panels (iv-vi) and panels (ix-xii)).

\subsection{Production and characterization of BoHV-4 recombinant strains expressing membrane anchored forms of IRAC I and IRAC II}

In order to test in vivo the potential of the IRAC membrane anchored forms described above as antigen candidates, we produced BoHV-4 recombinant strains expressing IRAC I CD46, -I HA, -II CD46 or -II HA under control of the hCMV IE promoter. The V. test BAC $\mathrm{G}$ plasmid was used as parental background. Resulting modified plasmids, called V. test BAC G IRAC I CD46, -I HA, -II CD46 or -II HA plasmids were checked by a combined 


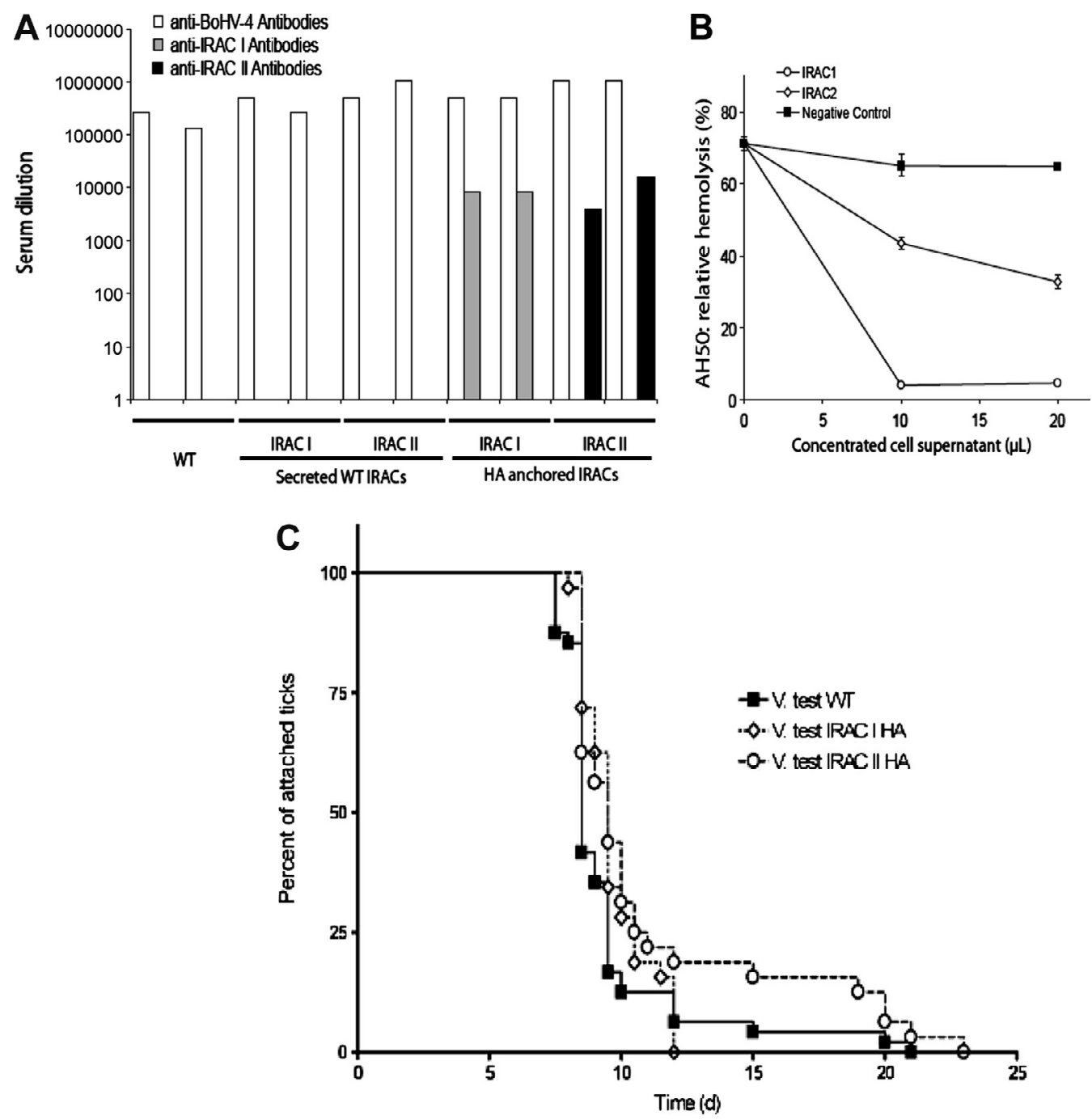

Figure 5. Infection by BoHV-4 strains expressing membrane anchored forms of IRAC elicits antibody response against IRAC. (a) Titres of anti-BoHV-4, anti-IRAC I and anti-IRAC II antibodies in sera from the two individual rabbits per group infected with the V. test strain of BoHV-4 or with the recombinant BoHV-4 V. test IRAC I, -IRAC II, -IRAC I HA or -IRAC II HA excised strains. Titres were evaluated for anti-BoHV-4, anti-IRAC I or anti-IRAC II antibodies by indirect immunofluorescent staining of CRFK cells transfected with plasmids expressing soluble WT IRAC. Serum titres are expressed as the reciprocal of the highest dilution of the serum that gave a positive signal on immunofluorescence (equivalent data were obtained in another experiment). (b) The ability of IRAC I and IRAC II to inhibit the complement alternative pathway of rabbit was tested using the $\mathrm{AH}_{50}$ assay as described in the methods. The data are expressed as the percentage of lysis observed when mock-infected concentrated cell supernatant was added to the test. (c) Effect of IRAC immunization on tick feeding. Female ticks were fed on vaccinated rabbits one month after the second immunization. Infested rabbits were checked daily. Anchored and detached ticks were counted. The number of anchored ticks (expressed as a percentage of the whole population) was plotted. 
HindIII restriction endonuclease-Southern blot approach (Figs. 1B and 1C), sequences of these plasmids were further confirmed by sequencing of the regions used to target recombination (data not shown). V. test BAC G IRAC I CD46, -I HA, -II CD46 and -II HA plasmids were then transfected into permissive EBL cells to reconstitute BoHV-4 V. test BAC G IRAC I CD46, -I HA, -II CD46 and -II HA recombinant strains, respectively. The molecular structure of the four recombinant strains was checked using the combined HindIII restriction endonuclease-Southern blot approach described above (Figs. 1B and 2C). The latter viruses were grown in EBL NLS-Cre to generate BoHV-4 V. test BAC G IRAC I CD46, -I HA, -II CD46 and -II HA excised strains in which the BAC cassette had been deleted. Knowing that the recombinants had the correct molecular structure, IRAC expression was tested (Fig. 3). Immunofluorescent staining of MDBK cells infected by BoHV-4 V. test BAC G IRAC I CD46, -I HA, -II CD46 and -II HA excised strains revealed that transgenes were expressed during the viral replication cycle at comparable levels to the ones observed for the recombinant viruses expressing secreted WT forms as shown on fixed and permeabilized cells. Staining of fixed cells demonstrated that IRAC membrane anchored forms were detectable at the surface of infected cells (Fig. 3, panels (v, vi, xi, xii)).

\subsection{Membrane anchored forms of IRAC proteins are detectable at the surface of PBMC infected ex vivo}

As BoHV-4 infects monocytes, we tested ex vivo whether infection of these cells with the different BoHV-4 recombinant strains described above leads to expression of IRAC proteins on the surface of infected cells. Detection of eGFP revealed that the tropism of BoHV-4 amongst PBMC is restricted to monocytes (Fig. 4, $\alpha$-CD11b panels). Interestingly, immunofluorescent staining of IRAC proteins demonstrated that eGFP positive cells also expressed IRAC I or II proteins on their surface. No specific fluorescence was detectable with WT infected cells (data not shown). Among
IRAC expressing cells, a proportion was negative for eGFP expression. This could be due to competition between the two hCMV promoters or due to differences in the sensitivity of the detection.

\subsection{Infection by BoHV-4 strains expressing membrane anchored forms but not soluble forms of IRAC elicits anti-IRAC antibody response}

Five groups consisting of two female New Zealand white rabbits were inoculated with BoHV-4 V. test WT or V. test BAC G IRAC I, V. test BAC G IRAC II, V. test BAC G IRAC I HA or V. test BAC G IRAC II HA excised recombinant strains. None of the animals developed fever or other clinical signs during the course of the experiment. Blood samples were collected one month after the second immunization and tested for anti-BoHV-4 and anti-IRAC I or II antibodies (Fig. 5A). All animals developed a comparable humoral immune response against BoHV-4 (Fig. 5A). While animals immunized with WT BoHV-4 or with BoHV-4 recombinants expressing soluble IRAC did not produced detectable anti-IRAC antibodies; rabbits infected with BoHV-4 recombinants expressing membrane anchored forms produced high titres of anti-IRAC specific antibodies (Fig. 5A). Interestingly, despite the relatively close homology existing between IRAC I and IRAC II (IRAC I and IRAC II amino-acid sequences are $66 \%$ identical), no cross-reactivity was detected between antiIRAC I and anti-IRAC II sera.

\subsection{Infection by BoHV-4 strains expressing membrane anchored forms of IRAC elicits an antibody response that does not affect markedly the blood feeding of adult ticks}

To determine if IRAC immunization could interfere with the tick feeding process on rabbit, we first investigated the property of both proteins to inhibit the alternative pathway of the rabbit complement (Fig. 5B) and then immunized animals were challenged one month after the second immunization with adult female I. ricinus ticks 
(Figs. 5C and 5D). The attachment rates were first examined during the initial $24 \mathrm{~h}$. No difference could be detected between the three groups where nearly all ticks attached. Blood meal durations were also compared between the three groups. On control rabbits, ticks accomplished their blood meal within 7-10 days. However, on BoHV-4 IRAC I HA and BoHV-4 IRAC II HA excised infected rabbits, ticks accomplished their blood meal within 8-12 days and 8-19 days, respectively (Fig. 5C). On BoHV-4 IRAC II HA excised vaccinated rabbits, $13.8 \%$ of ticks needed between 11 and 19 days to complete feeding and detach. On average, the blood meal duration was increased by approximately 1.5 day on BoHV-4 IRAC II HA excised infected rabbits $(10.17 \pm 2.81$ days $)$ as compared to controls $(8.74 \pm 1.15$ days $)$ $(p<0.05)$. There was no statistical weight difference detected between ticks from vaccinated or control rabbits (data not shown) and no difference in oviposition was monitored (data not shown). Finally, no difference in skin alteration at the feeding site was observed between vaccinated and control rabbits during the challenge and after the infestation period (data not shown).

\section{DISCUSSION}

Most attempts to develop protective vaccines against pathogens transmitted by ticks have met with little success [27]. The immunomodulatory molecules expressed in tick saliva could explain why these parasites transmit so many pathogens but also why vaccination against transmitted pathogens is hardly effective [22]. Vaccination against salivary immunomodulatory molecules could not only decrease the fitness of the arthropod but also protect the host from identified or unidentified tick transmitted pathogens. Recently, we cloned two different anti-complement proteins from the transcriptome of $I$. ricinus salivary glands [4] called IRAC I and II. Recombinant BoHV-4 vectors expressing these proteins were produced, however, our attempts to immunize rabbits against IRAC with these viruses invariably failed. In the present study, we produced BoHV-4 recombinants expressing membrane anchored forms of IRAC. Rabbit immunization with these recombinant viruses led to good antibody titres against IRAC I or II, however these antibodies only marginally affected tick blood feeding.

The theoretical framework used to describe the induction of humoral immune response is based on the "two signal model of lymphocyte activation" [1] that Bretsher and Cohn described more than 30 years ago [2]. According to this model, B-cell activation requires two signals. Signal one is provided by antigen binding to specific B-cell receptor, the second signal occurs when a primed antigen-specific CD4 + T cell engages with the antigen-specific B-cell through TCR/MHC interaction. Many studies have been dedicated to understanding this highly complex process. Some of them directly compared monovalent and oligovalent antigens in their efficiency to activate B-lymphocytes [14]. Their results suggest that oligomeric antigens are better than monomers to induce a humoral response. These observations hypothesis fit with the fact that intravenous administration of soluble antigens is often used to induce B-cell tolerance $[7,8]$.

Consistent with these observations and the model described above, we observed that immunization with BoHV-4 recombinants expressing transmembrane forms of IRAC led to a strong antibody response in rabbits contrary to viruses expressing soluble forms (Fig. 5A). This antibody response prolonged the feeding process of ticks significantly (Fig. 5C) but did not affect tick engorgement or oviposition rate. Lengthening of blood feeding is considered as the consequence of blood feeding disturbance by the host immune system and is therefore often seen as a positive result in tick vaccination. However, anti-tick vaccines need to induce rapid tick rejection in order to reduce transmission of tick-borne pathogens. From this point of view, vaccination against single IRAC molecules did not give better results than the ones obtained previously with other tick salivary antigens used separately [12, 13, 23].

Different hypothesis could explain the failure of the anti-IRAC immune response to interrupt the blood feeding process (Figs. 5B and 5C). Firstly, it is possible that the titre of the anti-IRAC antibodies produced is not high 
enough and/or that the isotypes expressed are not appropriate. Secondly, it is possible that efficient reject of the tick requires an immune response raised against both IRAC and eventually phylogenetically related molecules. Very recently, a group of 5 sequences homologous to IRAC was reported in I. ricinus [3]. When analysed with IRAC, the 5 new sequences (termed IxAC-B1 to 5) segregated into a relatively distant phylogenic group exhibiting around $40 \%$ amino-acid identity with the former sequences. IxAC-Bs like IRAC inhibit the alternative pathway of the complement system. These new sequences were shown to evolve, as we reported earlier for IRAC [4], by positive Darwinian selection. Couvreur et al. concluded that diversification of these sequences was associated with differences in antigenicity [3] based on the relatively low cross-reactivity of the sera raised against the different members. This hypothesis suggests that tick vaccination using IRAC homologues should involve several members covering the antigenic diversity of this anti-complement inhibitory protein family. Thirdly, one could postulate that efficient anti-tick vaccines should rely on several salivary antigens involved in the inhibition of different host physiological processes. To this end, some groups have identified the sialotranscriptome for different tick species $[15,19,29]$. These resources can now be used to identify other proteins with immunomodulatory activities that could be used as candidate antigens.

All together, the data presented in this study demonstrate that BoHV-4 has a potential as an in vivo expression vector. More importantly, the present study demonstrates that the immunogenicity of a soluble antigen can be greatly improved by anchoring it in membrane. In the future, this approach could be applied to other soluble antigens for which preliminary immunization attempts failed.

Acknowledgements. L. Gillet and B. Dewals are research associate and postdoctoral researcher of the "Fonds de la Recherche Scientifique - Fonds National Belge de la Recherche Scientifique" (FRS - FNRS), respectively. M. Thirion is a Research Fellow of the Belgian "Fonds pour la formation à la Recherche dans l'Industrie et dans l'Agriculture". The authors thank the Institute of Biology, University of Neufchâtel for providing the ticks and $\mathrm{C}$. Delforge for excellent and devoted technical assistance. This work was supported by the following grants: Région Wallonne (14628, 315543 and 0415703) and FNRS (FRSC 1.4.015. 04.F).

\section{REFERENCES}

[1] Baumgarth N., A two-phase model of B-cell activation, Immunol. Rev. (2000) 176:171-180.

[2] Bretscher P., Cohn M., A theory of self-nonself discrimination, Science (1970) 169:1042-1049.

[3] Couvreur B., Beaufays J., Charon C., Lahaye K., Gensale F., Denis V., et al., Variability and action mechanism of a family of anticomplement proteins in Ixodes ricinus, PLoS ONE (2008) 3:e1400.

[4] Daix V., Schroeder H., Praet N., Georgin J.P., Chiappino I., Gillet L., et al., Ixodes ticks belonging to the Ixodes ricinus complex encode a family of anticomplement proteins, Insect Mol. Biol. (2007) 16:155-166.

[5] Dewals B., Gillet L., Gerdes T., Taracha E.L., Thiry E., Vanderplasschen A., Antibodies against bovine herpesvirus 4 are highly prevalent in wild African buffaloes throughout eastern and southern Africa, Vet. Microbiol. (2005) 110:209-220.

[6] Donofrio G., Cavirani S., Simone T., van Santen V.L., Potential of bovine herpesvirus 4 as a gene delivery vector, J. Virol. Methods (2002) 101:49-61.

[7] Eynon E.E., Parker D.C., Small B cells as antigenpresenting cells in the induction of tolerance to soluble protein antigens, J. Exp. Med. (1992) 175:131-138.

[8] Gahring L.C., Weigle W.O., The induction of peripheral $\mathrm{T}$ cell unresponsiveness in adult mice by monomeric human gamma-globulin, J. Immunol. (1989) 143:2094-2100.

[9] Gillet L., Daix V., Donofrio G., Wagner M., Koszinowski U.H., China B., et al., Development of bovine herpesvirus 4 as an expression vector using bacterial artificial chromosome cloning, J. Gen. Virol. (2005) 86:907-917.

[10] Gillet L., Dewals B., Farnir F., de Leval L., Vanderplasschen A., Bovine herpesvirus 4 induces apoptosis of human carcinoma cell lines in vitro and in vivo, Cancer Res. (2005) 65:9463-9472.

[11] Hanon E., Vanderplasschen A., Lyaku S., Keil G., Denis M., Pastoret P.P., Inactivated bovine herpesvirus 1 induces apoptotic cell death of mitogen-stimulated bovine peripheral blood mononuclear cells, J. Virol. (1996) 70:4116-4120. 
[12] Imamura S., da Silva Vaz I. Jr., Sugino M., Ohashi K., Onuma M., A serine protease inhibitor (serpin) from Haemaphysalis longicornis as an antitick vaccine, Vaccine (2005) 23:1301-1311.

[13] Imamura S., Namangala B., Tajima T., Tembo M.E., Yasuda J., Ohashi K., Onuma M., Two serine protease inhibitors (serpins) that induce a bovine protective immune response against Rhipicephalus appendiculatus ticks, Vaccine (2006) 24:2230-2237.

[14] Kim Y.M., Pan J.Y., Korbel G.A., Peperzak V., Boes M., Ploegh H.L., Monovalent ligation of the B cell receptor induces receptor activation but fails to promote antigen presentation, Proc. Natl. Acad. Sci. USA (2006) 103:3327-3332.

[15] Lambson B., Nene V., Obura M., Shah T., Pandit P., Ole-Moiyoi O., et al., Identification of candidate sialome components expressed in ixodid tick salivary glands using secretion signal complementation in mammalian cells, Insect Mol. Biol. (2005) 14:403414.

[16] Lawrie C.H., Randolph S.E., Nuttall P.A., Ixodes ticks: serum species sensitivity of anticomplement activity, Exp. Parasitol. (1999) 93:207-214.

[17] Lawrie C.H., Sim R.B., Nuttall P.A., Investigation of the mechanisms of anti-complement activity in Ixodes ricinus ticks, Mol. Immunol. (2005) 42:31-38.

[18] Lopez O.J., Galeota J.A., Osorio F.A., Bovine herpesvirus type-4 (BHV-4) persistently infects cells of the marginal zone of spleen in cattle, Microb. Pathog. (1996) 21:47-58.

[19] Mans B.J., Andersen J.F., Francischetti I.M., Valenzuela J.G., Schwan T.G., Pham V.M., et al., Comparative sialomics between hard and soft ticks: Implications for the evolution of blood-feeding behavior, Insect Biochem. Mol. Biol. (2008) 38:42-58.

[20] Markine-Goriaynoff N., Gillet L., Karlsen O.A., Haarr L., Minner F., Pastoret P.P., et al., The core 2 beta-1,6-N-acetylglucosaminyltransferase-M encoded by bovine herpesvirus 4 is not essential for virus replication despite contributing to post-translational modifications of structural proteins, J. Gen. Virol. (2004) 85:355-367.

[21] Mejri N., Rutti B., Brossard M., Immunosuppressive effects of Ixodes ricinus tick saliva or salivary gland extracts on innate and acquired immune response of $\mathrm{BALB} / \mathrm{c}$ mice, Parasitol. Res. (2002) 88:192-197.

[22] Nuttall P.A., Labuda M., Tick-host interactions: saliva-activated transmission, Parasitology (2004) 129(Suppl.):S177-S189.

[23] Prevot P.P., Couvreur B., Denis V., Brossard M., Vanhamme L., Godfroid E., Protective immunity against Ixodes ricinus induced by a salivary serpin, Vaccine (2007) 25:3284-3292.

[24] Ribeiro J.M., Ixodes dammini: salivary anticomplement activity, Exp. Parasitol. (1987) 64:347353.

[25] Schroeder H., Daix V., Gillet L., Renauld J.C., Vanderplasschen A., The paralogous salivary anticomplement proteins IRAC I and IRAC II encoded by Ixodes ricinus ticks have broad and complementary inhibitory activities against the complement of different host species, Microbes Infect. (2007) 9:247-250.

[26] Thiry E., Bublot M., Dubuisson J., Van Bressem M.F., Lequarre A.S., Lomonte P., et al., Molecular biology of bovine herpesvirus type 4, Vet. Microbiol. (1992) 33:79-92.

[27] Titus R.G., Bishop J.V., Mejia J.S., The immunomodulatory factors of arthropod saliva and the potential for these factors to serve as vaccine targets to prevent pathogen transmission, Parasite Immunol. (2006) 28:131-141.

[28] Valenzuela J.G., Charlab R., Mather T.N., Ribeiro J.M., Purification, cloning, and expression of a novel salivary anticomplement protein from the tick, Ixodes scapularis, J. Biol. Chem. (2000) 275:1871718723.

[29] Valenzuela J.G., Francischetti I.M., Pham V.M., Garfield M.K., Mather T.N., Ribeiro J.M., Exploring the sialome of the tick Ixodes scapularis, J. Exp. Biol. (2002) 205:2843-2864.

[30] Wikel S.K., Acquired resistance to ticks: expression of resistance by $\mathrm{C} 4$-deficient guinea pigs, Am. J. Trop. Med. Hyg. (1979) 28:586-590.

[31] Zimmermann W., Broll H., Ehlers B., Buhk H.J., Rosenthal A., Goltz M., Genome sequence of bovine herpesvirus 4, a bovine Rhadinovirus, and identification of an origin of DNA replication, J. Virol. (2001) 75:1186-1194. 